\title{
RANKING DAS EMPRESAS DE TELECOMUNICAÇÕES E DE SEUS INDICADORES ECONÔMICOS FINANCEIROS UTILIZANDO-SE A ESTRATÉGIA MISTA DA TEORIA DOS JOGOS
}

\author{
Caroline Sulzbach Pletsch \\ Universidade Regional de Blumenau - FURB, SC, Brasil \\ carol_spletsch@yahoo.com.br
}

Alini da Silva

Universidade Regional de Blumenau - FURB, SC, Brasil

alinicont@gmail.com

Nelson Hein

Universidade Regional de Blumenau - FURB, SC, Brasil

hein@furb.br

Adriana Kroenke

Universidade Regional de Blumenau - FURB, SC, Brasil. Universidade Federal do Paraná UFPR, PR, Brasil

didlen@terra.com.br

\begin{abstract}
RESUMO
O objetivo deste estudo foi verificar o ranking das empresas de telecomunicações e de seus indicadores econômicos financeiros utilizando a estratégia mista da teoria dos jogos. Também, utilizou-se a correlação de Kendall para verificar alternância de posições no ranking das empresas e dos indicadores. Trata-se de um estudo descritivo, documental e quantitativo. Os resultados evidenciaram que houve alternância das posições das empresas, representando correlação não significante. Já a correlação dos indicadores foi forte e significante, por ter pouca alternância de posições. Os indicadores liquidez geral, retorno do investimento e liquidez corrente apresentaram-se como principais oponentes das empresas.
\end{abstract}

Palavras-chave: Teoria dos jogos - estratégias mistas - ranking - desempenho econômico financeiro.

\section{ABSTRACT}

The aim of this study was to determine the ranking of telecommunications and its financial economic indicators using a mixed strategy game theory companies. Also, we used the Kendall correlation to verify alternation of positions in the ranking of companies and indicators. This is a descriptive, document and quantitative study. The results showed that there were alternating the positions of companies, representing no significant correlation. Have the correlation of indicators was high and significant, to have little change of positions. The general liquidity indicators, return on investment and current liquidity presented themselves as leading opponents of companies.

Keywords: Game Theory - mixed strategies - ranking - financial and economic performance.

\section{INTRODUÇÃO}

A teoria dos jogos ao longo dos anos vem abrangendo diversas áreas, tanto matemática, econômica ou social. Para Abbade (2009), a teoria dos jogos engloba problemas 
de decisão de seus participantes ou jogadores, a possível previsão dos comportamentos destes, e os resultados a serem alcançados.

Um jogo, segundo Fiani (2006, p. 12) pode ser conceituado como "situações que envolvam interações entre agentes racionais que se comportam estrategicamente [...]". Os jogadores ao tomarem decisões, consideram os efeitos que podem repercutir nos demais jogadores, bem como a reação dos mesmos quanto a esta decisão (FIANI, 2006).

De acordo com Myerson (2013), a teoria dos jogos fornece técnicas matemáticas para análise de decisões que duas ou mais pessoas tomam e que influenciam o bem estar do outro indivíduo. Trata-se de modelos matemáticos de conflito e interação entre as decisões estratégicas.

A teoria dos jogos no contexto empresarial tem como finalidade demonstrar aos gestores como melhorar o relacionamento com os usuários externos (LOZANO, 2011). Desta forma, os indicadores econômicos financeiros são importantes para melhorar o relacionamento entre os usuários externos e a empresa, por repassar informações sobre o desempenho da mesma. Assim, a organização apresenta-se como único jogador e seu oponente é contra a natureza, considerados os indicadores econômicos financeiros.

Jogos contra a natureza, segundo Kreuzberg (2013, p. 76) "ao contrário dos jogos de duas pessoas-soma zero [...] são também denominados por jogos de uma pessoa só, pois nesses casos a natureza seria o outro jogador". Em um jogo de uma pessoa, a decisão a ser tomada concentra-se em apenas um centro de decisões, com condições de decidir entre as alternativas existentes sobre determinado cenário (SOUZA, 2003).

Neste contexto, apresenta-se a questão de pesquisa: Qual o ranking das empresas de telecomunicações e de seus indicadores econômicos financeiros utilizando-se a estratégia mista da teoria dos jogos? Para responder esta questão, o estudo tem como objetivo verificar o ranking das empresas de telecomunicações e de seus indicadores econômicos financeiros utilizando a estratégia mista da teoria dos jogos.

Estudos, tais como de Silva e Cordeiro Filho (2010); Alencar et al. (2010); Kreuzberg (2013); Kroenke et al. (2013) utilizaram a teoria dos jogos em suas pesquisas. Silva e Cordeiro Filho (2010) verificaram a aplicação de conceitos da teoria dos jogos nos principais periódicos internacionais da área contábil, no período de 2005 a 2009. Concluíram que há ausência de pesquisas em contabilidade utilizando a teoria dos jogos, o que indica a oportunidade para novas pesquisas.

Alencar et al. (2010) analisaram os processos de tomada de decisão estratégica de nível corporativo que resultou na fusão da Sadia com a Perdigão, por meio da teoria dos jogos. Os resultados demonstram que havia dois jogos diferentes, um contra a fusão e outro a favor, permitindo ainda observar a lógica das decisões e sua racionalidade, em ambas as situações, a realidade foi coerente com a teoria, ou vice versa.

No estudo de Kroenke et al. (2013) foi identificado os artigos publicados em eventos científicos na área da Administração e Contabilidade, no período de 2007 a 2012, referentes a teoria dos jogos. Foi concluído com base nos artigos analisados, que há poucos estudos sobre teoria dos jogos.

Kreuzberg (2013) utilizou a estratégia mista da teoria dos jogos, para realizar um cenário contra a natureza entre empresas e seus indicadores econômicos e sociais. Os resultados mostraram que os indicadores de rentabilidade e de mercado foram os que proporcionaram maiores ganhos para as empresas e foram os mais perigosos, conforme a teoria dos jogos.

O presente estudo justifica-se devido à falta de estudos na área contábil envolvendo a teoria dos jogos, tornando-se válidos outros estudos para aprofundar o conhecimento desta teoria. Destaca-se a importância de estratégias da teoria dos jogos no meio empresarial, visto sua amplitude racional em tomadas de decisões, auxiliando o gestor em escolhas mais acertadas e que também se baseia em estratégias de seu oponente. 


\section{TEORIA DOS JOGOS E ESTRATÉGIAS MISTAS}

A teoria dos jogos teve seu início com a publicação do trabalho "Theory of Games and Economic Behavior” em 1944 pelo matemático John Von Neumann, juntamente com o economista Oskar Morgenstern (DAVIS, 1973).

Para determinar estratégias de causas e consequências em um determinado fato, a teoria dos jogos auxilia o jogador, utilizando sua racionalidade humana para determinar suas decisões (MCCAIN, 2008).

Segundo Lins e Calôba (2006), a teoria dos jogos pode ser implantada em determinada estratégia, conflito ou disputa, a fim de se chegar à solução mais ótima possível. Para se chegar à solução mais ótima possível pode-se utilizar a estratégia pura ou a estratégia mista. seguintes:

Os elementos que compõe a formulação de um jogo em sua forma normal são os

(a) Um jogo finito de estratégias puras $E_{1}=\left\{I_{1}, I_{2}, \ldots, I_{m}\right\}$, para um jogador I e um conjunto finito de estratégias puras para um jogador II, $E_{2}=\left\{I I_{1}, I I_{2}, \ldots, I I_{n}\right\}$.

(b) Uma matriz real de ordem $m \times n, A=\left(a_{i j}\right)$. Cada elemento desta matriz $a_{i j}$ é o pagamento para o jogador $I$ quando este elege a estratégia $I_{i}$ e o jogador II escolhe a estratégia $I I_{j}$. O pagamento para o jogador nestas condições é $-a_{i j}$.

Esta modelagem, guardadas as devidas proporções, é encontrada com simbologia similar nos trabalhos seminais de Blackwell e Girshick (1954), Dresher, Shapley e Tucker (1957), Dresher, Tucker e Wolfe (1964), Gale (1960), Harsanyi (1977), Karlin (1959), Kuhn e Tucker (1950, 1953), Luce e Raiffa (1957), Mc Kinsey (1952), Owen (1968), Parthasarathy e Raghavan (1971), Rapoport (1966, 1970), Shubik (1959, 1980), Tucker e Luce (1959), Von Neumann e Morgenstern (1944, 1953) e Zeleny (1982).

A solução (ou soluções) de um jogo bipessoal de soma-zero pode ser caracterizada de duas formas: mediante as estratégias de segurança (maximin) e com o conceito de ponto de equilíbrio.

Em jogos de soma-zero quando um jogador objetiva maximizar seu pagamento, está tentando minimizar o pagamento de seu oponente, por exemplo. Cada jogador considera o pior resultado que pode conseguir com cada uma de suas estratégias e depois elege a estratégia que lhe proporciona o melhor entre os piores resultados. Segundo Hein, Kroenke e Faria (2009), para cada estratégia pura $I_{i} e E_{1}$, o nível de segurança do jogador I é o pagamento que pode ser assegurado com a estratégia, independente das ações do jogador II.

$$
v_{I}\left(I_{i}\right)=\min _{j} a_{i j}
$$

De similar modo: para cada estratégia pura $I I_{j} \in E_{2}$, o nível de segurança do jogador II é o pagamento que é assegurado com esta estratégia, independentemente das ações do jogador I.

$$
v_{I I}\left(I I_{j}\right)=\max _{i} a_{i j}
$$

Hein et al. (2009) agregam que o valor minimax (o valor inferior do jogo) do jogador $I$ é:

$$
v_{I}=\max _{i} v_{I}\left(I_{i}\right)=\max _{i} \min _{j} a_{i j}
$$

Uma estratégia de segurança, ou estratégia maximin é a que proporciona ao jogador seu valor maximin. $\mathrm{O}$ valor minimax (ou valor superior do jogo) do jogador II.

$$
v_{I I}=\min _{j} v_{I I}\left(I I_{j}\right)=\min _{j} \max _{i} a_{i j}
$$

Uma estratégia de segurança, ou estratégia minimax é a que proporciona ao jogador seu valor minimax. Singleton e Tyndall (1977) destacam isso por meio de um teorema: para cada jogo matricial da matriz $A=\left(a_{i j}\right)$ se verifica:

(i) Os valores $v_{I}$ e $v_{I I}$ são únicos;

(ii) Existe ao menos uma estratégia de segurança para cada jogador;

(iii) $v_{I} \leq v_{I I}$. 
Assim, um jogo matricial $A=\left(a_{i j}\right)$ possui um ponto de sela quando se verifica:

$$
v_{I}=v_{I I}
$$

Este valor comum se denomina valor do jogo e é dado pelo menor elemento de sua linha e o máximo de sua coluna e é denotado por V. Um ponto de sela, se existir, terá como pagamento um par de estratégias de segurança. Estas estratégias, juntamente com o valor do jogo, constituem a solução do jogo.

As estratégias que proporcionam os pontos de sela não têm porque serem únicas. Se existe mais de um par então são equivalentes, ou seja, proporcionam o mesmo valor do jogo (V). Entretanto, nem todos os jogos de soma nula possuem um ponto de sela definido por estratégias puras (GROSSMAN, 1992).

Neste caso, usam-se estratégias mistas, selecionando aleatoriamente as estratégias, mesclando-as de acordo com alguma distribuição de probabilidades no conjunto de estratégias puras do jogador.

\section{PROCEDIMENTOS METODOLÓGICOS}

O presente estudo caracteriza-se como descritivo, documental e quantitativo. Descritivo por descrever a posição das empresas de telecomunicações e de seus indicadores econômicos financeiros nos rankings formados pela estratégia mista da teoria dos jogos. É documental pela busca de informações em bases de dados. A pesquisa é quantitativa, ao utilizar métodos estatísticos para o tratamento dos dados.

\subsection{População e amostra}

A população do presente estudo é composta por todas as empresas listadas na Bolsa de Valores, Mercadorias e Futuros de São Paulo - BM\&F Bovespa. A amostra, por sua vez, compreendeu as empresas do setor de telecomunicações, totalizando 10 empresas. A amostra final do estudo foi composta por 9 empresas, decorrente da disponibilidade de informações na base de dados utilizada.

\subsection{Coleta e análise dos dados}

Os dados foram coletados na base de dados Economática ${ }^{\circledR}$, em que se buscaram os indicadores econômicos financeiros das empresas analisadas no período de 2008 a 2012 . Os quais podem ser verificados no quadro 1 .

Quadro 1 - Indicadores econômicos financeiros

\begin{tabular}{|c|c|c|}
\hline Indicadores & Descrição & Autores \\
\hline Liquidez Geral & (Ativo Circulante + Ativo não circulante) / (Passivo Circulante + \\
Passivo Não Circulante) & $\begin{array}{c}\text { Bezerra e Corrar } \\
(2006) .\end{array}$ \\
\hline ROI & Lucro Líquido / Ativo Total & Bastos et al. (2009). \\
\hline $\begin{array}{c}\text { Liquidez } \\
\text { Corrente }\end{array}$ & Ativo Circulante / Passivo Circulante & $\begin{array}{c}\text { Bortoluzzi et al. } \\
(2011) .\end{array}$ \\
\hline ROE & Lucro Líquido / Patrimônio Líquido & $\begin{array}{c}\text { Bortoluzzi et al. } \\
(2011) .\end{array}$ \\
\hline Capital de Giro & Ativo Circulante - Passivo Circulante & $\begin{array}{c}\text { Sanvicente e Minardi } \\
(1998) .\end{array}$ \\
\hline Fluxo de Caixa & Fluxo de caixa operacional + Fluxo de Caixa de Investimentos + \\
Fluxo de Caixa de Financiamento & Bastos et al. (2009). \\
\hline Endividamento & Endividamento Total / Patrimônio Líquido & $\begin{array}{c}\text { Bezerra e Corrar } \\
(2006) .\end{array}$ \\
\hline ROA & Lucro Líquido / Ativo total & $\begin{array}{c}\text { Bortoluzzi et } \\
\text { al.(2011) }\end{array}$ \\
\hline
\end{tabular}

Fonte: Dados da pesquisa.

Para a análise dos dados, foi utilizada a estratégia mista da teoria dos jogos para formar os rankings das empresas de telecomunicações, bem como para verificar os 
indicadores econômicos financeiros que possuem maior influência no desempenho das organizações.

Kreuzberg (2013) organizou o ranqueamento de um conjunto de empresas, em que a determinação das estratégias mistas serviu como o posicionamento contábil. A versão desenvolvida por Kreuzberg (2013) lidou com jogos escalares. Uma estratégia mista para um jogador é uma distribuição de probabilidade no conjunto de suas estratégias puras.

Tipicamente um jogador possui $n$ estratégias puras. Uma estratégia mista para ele é uma n-upla $x=\left(x_{1}, x_{2}, \ldots, x_{n}\right)$ tal que $\sum_{i=1}^{n} x_{i}=1,0 \leq x_{i} \leq 1$, onde $x_{i}$ indica a probabilidade com que o jogador selecionará sua i-ésima estratégia pura.

O conjunto de estratégias mistas sempre inclui todas as estratégias puras, porque estas últimas podem ser consideradas como um caso especial de estratégia mista em que a correspondente estratégia pura joga com probabilidade um e todas as demais com probabilidade zero.

Seja $A=\left(a_{i j}\right), 1 \leq i \leq n ; 1 \leq j \leq m$ a matriz de pagamentos do jogo. Sejam X e Y os conjuntos de estratégias mistas dos jogadores I e II, respectivamente:

$$
\begin{aligned}
& X=\left\{x \in \mathbb{R}^{m} / \sum_{i=1}^{n} x_{i}=1 ; x_{i} \geq 0, i=1,2, \ldots, n\right\} \\
& Y=\left\{y \in \mathbb{R}^{n} / \sum_{j=1}^{m} y_{i}=1 ; y_{i} \geq 0, j=1,2, \ldots, m\right\}
\end{aligned}
$$

Para analisar o resultado do jogo, quando um (ou ambos) jogador (es) utilizam estratégias mistas, pode-se utilizar o conceito de valor esperado, neste caso a função de pagamentos do jogo é:

$$
v(x, y)=\sum_{i=1}^{n} \sum_{j=1}^{m} x_{i} a_{i j} y_{j} ; x \in X, y \in Y
$$

Onde, $v(x, y)$ é o valor esperado em conseguir os pagamentos do jogo com a combinação das estratégias mistas $x \in X$ e $y \in Y$. Para cada estratégia mista $x \in X$, o nível de segurança do jogador I é o valor esperado que possa ser assegurado com essa estratégia, independente das ações do jogador II.

$$
v_{I}(x)=\min _{y \in Y} v(x, y)
$$

De similar modo, para cada estratégia mista $y \in Y$, o nível de segurança do jogador II é o valor esperado que possa assegurar essa estratégia, independente das ações do jogador I.

$$
v_{I I}(y)=\max _{x \in X} v(x, y)
$$

O valor maximin dado pelas estratégias mistas do jogador I é:

$$
v_{I}^{M}=\max _{x \in X} \min _{y \in Y} v(x, y)
$$

Uma estratégia de segurança ou estratégia maximin é a que proporciona ao jogador seu valor maximin.

O valor minimax dado pelas estratégias mistas do jogador II é:

$$
v_{I I}^{M}=\min _{y \in Y} \max _{x \in X} v(x, y)
$$

De mesmo modo uma estratégia de segurança ou estratégia minimax é a que proporciona ao jogador seu valor minimax. Em um jogo matricial de soma-zero se verifica:

(i) Os valores $v_{I}^{M}$ e $v_{I I}^{M}$ são únicos;

(ii) Existe ao menos uma estratégia mista de segurança para cada jogador;

(iii) Os níveis de segurança dados em estratégias puras e mistas verificam: $v_{I} \leq v_{I}^{M}$ e $v_{I I} \leq v_{I I}^{M}$.

As estratégias mistas $x^{*} \in X$ e $y^{*} \in Y$ são ótimas para os jogadores I e II respectivamente, se: 


$$
\begin{gathered}
v_{I}^{M}=\min _{y \in Y} v\left(x^{*}, y\right)=\min _{y \in Y} x^{* t} A y \\
v_{I I}^{M}=\max _{x \in X} v\left(x, y^{*}\right)=\max _{x \in X} x^{t} A y^{*}
\end{gathered}
$$

O nível de segurança para uma estratégia mista $\hat{x} \in X$ vem dado por $v_{I}(\hat{x})=$ $\min _{y \in Y} \hat{x}^{t} A y$, cuja valoração pode ser obtida por meio do problema dual anterior:

$$
\operatorname{Max} \lambda(\hat{x})
$$

Sujeito a: $e \lambda(\hat{x}) \leq \hat{x}^{t} A$

$$
\hat{x} \in X, \lambda(\hat{x}) \in \mathbb{R}
$$

Sendo $e=(1, \ldots, 1)^{t}$. As estratégias que proporcionam os melhores níveis de segurança são as que verificam $v_{I}^{M}=\max _{x \in X} v_{I}(x)$. Estas estratégias, assim como o valor do jogo podem ser obtidas por meio do problema de programação linear:

Sujeito a: $e v_{I} \leq x^{t} A$

\section{$\operatorname{Max} v_{I}$}

$x \in X$

Pode-se assumir o mesmo raciocínio para o jogador II. Ao minimizar seu nível de segurança de forma que limite o outro jogador, chega-se a outro problema de programação linear:

Sujeito a: $A y \leq v_{I I} e$

\section{$\operatorname{Min} v_{I I}$}

$$
y \in Y
$$

Comparando-se as duas formulações, verifica-se que são duais com soluções ótimas $x^{*}$ e $y^{*}$. Então $v_{I}^{*}=v_{I I}^{*}$, ou seja, as estratégias se autolimitam. Isso é conhecido pela denominação de Teorema Minimax. Este teorema é enunciado: em todo jogo bipessoal finito de soma-zero, existem estratégias ótimas $x^{*} \in X, y^{*} \in Y$, para cada jogador e verifica-se $v_{I}^{M}=v_{I I}^{M}=v^{*}$, sendo $v^{*}$, o valor do jogo.

Este resultado põe de manifesto que as estratégias de segurança ótimas não só otimizam os níveis de segurança de cada jogador, mas também limitam os pagamentos do oponente. O teorema minimax foi demonstrado por Von Neumann (1928) e posteriormente foram elaboradas diversas demonstrações, entre as quais se destaca a de Kakutani (1941), que empregou o teorema do ponto fixo de Brouwer.

Às vezes as estratégias de um ou mais jogadores estão submetidas a restrições adicionais, dando lugares aos denominados jogos restringidos. Estes tipos de jogos permitem uma formulação mais realista e prática de certos problemas de decisão sob incerteza. Assim, um jogador pode incorporar ao seu conjunto de estratégias, restrições que representam limitações de recursos, relações técnicas ou considerar uma possível informação que um jogador possui a respeito da frequência relativa com que seu oponente utiliza suas estratégias.

Charnes (1963) estabeleceu a equivalência entre certos problemas lineares e os jogos matriciais nos quais as estratégias mistas estão submetidas a restrições lineares. Em alguns casos particulares, o conjunto de restrições adicionais pode ser representado em função de seus pontos extremos, o que permite o tratamento do problema em termos de um jogo transformado (FERNÁNDEZ; MONROY; PUERTO, 1998).

Uma das propriedades das estratégias ótimas dos jogos matriciais ocorre quando ambos jogadores as utilizam, nenhum deles se beneficia se trocar para outra estratégia, enquanto que se a mantiver, se mantém ótima.

Quando os jogadores I e II se mantiverem em suas estratégias ótimas, caso o jogador II siga jogando $y^{*}$ e o jogador I troque para outra estratégia $x$, este irá piorar sua situação, ou seja, se o problema for de lucros, este baixará, já se for de custos, estes aumentarão. O mesmo vale para o jogador II, caso este deixe a estratégia $y^{*}$ e o jogador I se mantenha na estratégia ótima.

As estratégias $x^{*}$ e $y^{*}$ formam um ponto de equilíbrio. Um par de estratégias $x^{*} \in X$ e $y^{*} \in Y$ é um ponto de equilíbrio para um jogo matricial A se: 
Ou ainda:

$$
v\left(x, y^{*}\right) \leq v\left(x^{*}, y^{*}\right) \leq v\left(x^{*}, y\right), \forall x \in X, \forall y \in Y
$$

$$
x^{t} A y \leq x^{* t} A y \leq x^{* t} A y \quad \forall x \in X, \forall y \in Y
$$

A primeira desigualdade estabelece que $x^{*}$ é melhor resposta ao jogador I, para a estratégia $y^{*}$ do jogador II. A segunda estabelece que $y^{*}$ é a melhor resposta ao jogador II, para a estratégia $x^{*}$ do jogador I.

É possível a ocorrência de que um jogo matricial tenha mais de um ponto de equilíbrio, porém neste caso são equivalentes e podem ser combinados entre si para formar um novo ponto de equilíbrio, proporcionando os mesmos pagamentos.

Em jogos de soma-zero, os conceitos de solução, estratégias ótimas e pontos de equilíbrio são equivalentes. Sejam $x^{*} \in X$ e $y^{*} \in Y$ um par de estratégias de um jogo matricial, $\left(x^{*}, y^{*}\right)$, é um ponto de equilíbrio do jogo se, e somente se, $\left(x^{*}, y^{*}, v^{*}\right)$ é uma solução do jogo.

Este resultado estabelece que as estratégias ótimas formam pares de estratégias em equilíbrio e são os únicos pontos de equilíbrio. Este teorema pode ser interpretado em termos de solução de um jogo. Hein et al. (2009) afirmam: se um jogo possui mais de uma solução, todas proporcionam o mesmo valor do jogo.

Quando estes resultados são generalizados para jogos de $n$ pessoas, com $n>2$, ou ainda para jogos de soma não-nula, as propriedades das estratégias de equilíbrio em serem equivalentes se perdem. Assim, um par de estratégias maximin não é necessariamente um par de estratégias em equilíbrio, ou vice-versa. Todos os pontos de equilíbrio não proporcionam necessariamente o mesmo pagamento. Portanto, não há um conceito único de solução do jogo.

Outro problema que surge é ligado ao conceito de estratégia mista e sua relevância quando o jogo é jogado uma só vez. Isto deriva do fato de que a noção de pagamento esperado parece aplicável em um jogo repetido várias vezes. Porém, em um jogo que se joga uma só vez, pode não ter sentido escolher uma estratégia de acordo com a distribuição de probabilidade associada. Contudo, a probabilidade associada a cada estratégia é uma indicação a ser seguida em cenários futuros, e mesmo não existindo sequência no jogo, pode ser interpretada como sendo um esquema de preferências a serem seguidas, ou seja, a formação de um ranking entre as mesmas.

Neste artigo, as empresas compõem as opções do jogador I e seus indicadores as opções do jogador II. O ranqueamento de empresas (primal) e de seus indicadores por meio de jogos escalares foi desenvolvido por Kreuzberg (2013). A análise desenvolvida está inserida naquilo que é denominado jogos matriciais vetoriais, nos quais os pagamentos que os jogadores recebem vêm representados por vetores, no lugar de números reais. Apresenta-se a seguir na tabela 1, o cenário do jogo com os indicadores econômicos financeiros do ano de 2008 e as empresas correspondentes, a fim de demonstrar o modelo utilizado nos demais cenários para a realização do ranking das empresas. Já para a formação do ranking dos indicadores econômicos financeiros trocou-se a coluna das empresas pelos indicadores e a coluna dos indicadores pelas empresas.

Tabela 1 - Cenário do jogo para a formação do ranking das empresas do ano de 2008.

\begin{tabular}{l|c|c|c|c|c|c|c|c}
\hline Empresa & ROA & ROE & ROI & Liq_Geral & Liq_Cor & FC & Cap Giro & Endiv. \\
\hline Embratel Part & 4,083 & 7,259 & 0,040 & 0,768 & 0,894 & 7,244 & 6,979 & 8,687 \\
\hline Jereissati & 1,916 & 6,462 & 0,002 & 0,725 & 1,91 & 9,181 & 7,077 & 8,682 \\
\hline La Fonte Tel & 1,302 & 5,650 & $-0,006$ & 0,700 & 1,885 & 9,257 & 7,070 & 8,681 \\
\hline LF Tel & $-0,472$ & $-1,914$ & $-0,005$ & 0,711 & 1,883 & 9,241 & 7,069 & 8,681 \\
\hline Oi & 5,818 & 16,486 & 0,058 & 0,886 & 1,281 & 9,019 & 7,055 & 8,683 \\
\hline Telef Brasil & 12,105 & 24,090 & 0,121 & 0,801 & 1,105 & 8,981 & 7,026 & 8,686 \\
\hline Telemar & 3,488 & 12,552 & 0,028 & 0,74 & 1,842 & 9,791 & 7,256 & 8,682 \\
\hline Inepar Tel & $-236,228$ & 8,100 & $-2,362$ & 0,017 & 0,062 & 8,176 & 7,000 & 8,565 \\
\hline
\end{tabular}




\begin{tabular}{l}
\hline Tim Part S/A \\
\hline Fonte: Dados da pesquisa.
\end{tabular}

Com base neste cenário, apresenta-se o modelo aplicado para a formação dos rankings. Da mesma forma, foi realizado nos demais anos para a formação dos rankings de empresas e também para a formação dos rankings nos anos de 2008 a 2012 dos indicadores econômicos financeiros.

Sujeito a:

$$
\operatorname{Max} Z=v
$$

$$
\begin{gathered}
4,083 x_{1}+1,916 x_{2}+1,302 x_{3}+\cdots+1,109 x_{9} \geq v \\
7,259 x_{1}+6,462 x_{2}+5,650 x_{3}+\cdots+2,312 x_{9} \geq v \\
\vdots \quad \vdots \quad \ddots \quad \vdots \quad \vdots \\
8,687 x_{1}+8,682 x_{2}+8,681 x_{3}+\cdots+8,685 x_{9} \\
x_{1}+x_{2}+x_{3}+\cdots+x_{9}=1 \\
x_{1}, x_{2}, x_{3}, \ldots, x_{9} \geq 0
\end{gathered}
$$

Os resultados do modelo aplicado na forma de problemas de programação linear (PPLs) são apresentados na seção descrição e análise dos dados.

Ao final, foi utilizada ainda, a correlação de Kendall por meio do programa SPSS $®$, para verificar se as empresas e os indicadores tiveram alternância em suas posições nos rankings (em que estes se originaram pelas estratégias mistas) dos períodos analisados.

\section{DESCRIÇÃO E ANÁLISE DOS DADOS}

Apresenta-se nesta seção a análise do ranking das empresas de telecomunicações e dos indicadores econômicos financeiros gerados pela estratégia mista da teoria dos jogos, bem como análise da correlação de Kendall de ambos. Na tabela 2, apresenta-se o ranking das empresas de telecomunicações.

Tabela 2 - Ranking das empresas de telecomunicações

\begin{tabular}{l|c|c|c|c|c}
\hline \multicolumn{1}{c|}{ Empresa } & $\mathbf{2 0 0 8}$ & $\mathbf{2 0 0 9}$ & $\mathbf{2 0 1 0}$ & $\mathbf{2 0 1 1}$ & $\mathbf{2 0 1 2}$ \\
\hline LF Tel. & 1 & 4 & 8 & 1 & 1 \\
\hline Tim Partic. & 2 & 6 & 3 & 5 & 8 \\
\hline Telef Brasil & 3 & 8 & 2 & 3 & 6 \\
\hline OI & 4 & 1 & 4 & 6 & 9 \\
\hline Embratel Part. & 5 & 7 & 1 & 2 & 4 \\
\hline Telemar & 6 & 3 & 5 & 8 & 7 \\
\hline Jereissati & 7 & 5 & 7 & 7 & 3 \\
\hline Inepar Tel. & 8 & 9 & 6 & 9 & 5 \\
\hline La Fonte Tel. & 9 & 2 & 9 & 4 & 2 \\
\hline Font Das & &
\end{tabular}

Fonte: Dados da pesquisa

Observa-se na tabela 2 a posição que as empresas ocuparam nos rankings no período de 2008 a 2012. Notam-se as seguintes posições para as empresas, considerando a ordem crescente dos anos: LF telecomunicações: $1^{\mathrm{a}}, 4^{\mathrm{a}}, 8^{\mathrm{a}}, 1^{\mathrm{a}}, 1^{\mathrm{a}}$, Tim Participações: $2^{\mathrm{a}}, 6^{\mathrm{a}}, 3^{\mathrm{a}}, 5^{\mathrm{a}} \mathrm{e}$ $8^{\mathrm{a}}$; Telef Brasil: $3^{\mathrm{a}}, 8^{\mathrm{a}}, 2^{\mathrm{a}}, 3^{\mathrm{a}}$ e $6^{\mathrm{a}}$; Oi: $4^{\mathrm{a}}, 1^{\mathrm{a}}, 4^{\mathrm{a}}, 6^{\mathrm{a}}$ e $9^{\mathrm{a}}$; Embratel Participações: $5^{\mathrm{a}}, 7^{\mathrm{a}}, 1^{\mathrm{a}}, 2^{\mathrm{a}}$ e

\begin{tabular}{|c|c|c|c|c|c|c|}
\hline & & 2008 & 2009 & 2010 & 2011 & 2012 \\
\hline \multirow{2}{*}{2008} & Correlações de coeficiente & 1,000 & 0,000 & 0,333 & 0,444 & $-0,222$ \\
\hline & Sig. ( 2 extremidades $)$ & - & 1,000 & 0,211 & 0,095 & 0,404 \\
\hline 2009 & Correlações de coeficiente & 0,000 & 1,000 & $-0,333$ & 0,000 & 0,000 \\
\hline
\end{tabular}
$4^{\mathrm{a}}$; Telemar: $6^{\mathrm{a}}, 3^{\mathrm{a}}, 5^{\mathrm{a}}, 8^{\mathrm{a}}$ e $7^{\mathrm{a}}$; Jereissati: $7^{\mathrm{a}}, 5^{\mathrm{a}}, 7^{\mathrm{a}}, 7^{\mathrm{a}}$ e $3^{\mathrm{a}}$; Inepar Telecomunicações: $8^{\mathrm{a}}, 9^{\mathrm{a}}, 6^{\mathrm{a}}$, $9^{\mathrm{a}}$ e $5^{\mathrm{a}}$; La Fonte Telecomunicações: $9^{\mathrm{a}}, 2^{\mathrm{a}}, 9^{\mathrm{a}}, 4^{\mathrm{a}} 2^{\mathrm{a}}$.

Com o intuito de verificar a alternância das posições das empresas no período analisado, aplicou-se a correlação de Kendall, a qual se apresenta na tabela 3.

Tabela 3 - Correlação do ranking das empresas de telecomunicações 


\begin{tabular}{l|l|c|c|c|c|c}
\hline & Sig. (2 extremidades) & 1,000 & - & 0,211 & 1,000 & 1,000 \\
\hline \multirow{2}{*}{2010} & Correlações de coeficiente & 0,333 & $-0,333$ & 1,000 & 0,222 & $-0,444$ \\
\cline { 2 - 6 } & Sig. (2 extremidades) & 0,211 & 0,211 & - & 0,404 & 0,095 \\
\hline \multirow{2}{*}{2011} & Correlações de coeficiente & 0,444 & 0,000 & 0,222 & 1,000 & 0,333 \\
\cline { 2 - 6 } & Sig. (2 extremidades) & 0,095 & 1,000 & 0,404 & - & 0,211 \\
\hline \multirow{2}{*}{2012} & Correlações de coeficiente & $-0,222$ & 0,000 & $-0,444$ & 0,333 & 1,000 \\
\cline { 2 - 6 } & Sig. (2 extremidades) & 0,404 & 1,000 & 0,095 & 0,211 & - \\
\hline \multirow{2}{*}{ F }
\end{tabular}

Fonte: Dados da pesquisa

A tabela 3 demonstra que não houve significância em nenhum período da análise, ou seja, houve grande alternância nas posições das empresas nos rankings de 2008 a 2012 . A tabela 4 demonstra o ranking dos indicadores econômicos financeiros.

Tabela 4 - Ranking dos indicadores econômicos financeiros

\begin{tabular}{l|c|c|c|c|c}
\hline \multicolumn{1}{c|}{ Indicadores } & $\mathbf{2 0 0 8}$ & $\mathbf{2 0 0 9}$ & $\mathbf{2 0 1 0}$ & $\mathbf{2 0 1 1}$ & $\mathbf{2 0 1 2}$ \\
\hline Liquidez Geral & 1 & 1 & 2 & 2 & 4 \\
\hline ROI & 2 & 2 & 1 & 1 & 2 \\
\hline Liquidez Corrente & 3 & 3 & 3 & 3 & 3 \\
\hline ROE & 4 & 6 & 5 & 4 & 5 \\
\hline Capital de Giro & 5 & 4 & 4 & 5 & 6 \\
\hline Fluxo de Caixa & 6 & 5 & 6 & 6 & 8 \\
\hline Endividamento & 7 & 8 & 7 & 7 & 7 \\
\hline ROA & 8 & 7 & 8 & 8 & 1 \\
\hline
\end{tabular}

Fonte: Dados da pesquisa

Verifica-se na tabela 4, a posição que os indicadores econômicos financeiros ocuparam nos rankings no período de 2008 a 2012. Notam-se as seguintes posições dos indicadores econômicos financeiros, considerando a ordem crescente dos anos: Liquidez Geral: $1^{\mathrm{a}}, 1^{\mathrm{a}}, 2^{\mathrm{a}}, 2^{\mathrm{a}}$ e $4^{\mathrm{a}}$; ROI: $2^{\mathrm{a}}, 2^{\mathrm{a}}, 1^{\mathrm{a}}, 1^{\mathrm{a}}$ e $2^{\mathrm{a}}$; Liquidez Corrente: $3^{\mathrm{a}}, 3^{\mathrm{a}}, 3^{\mathrm{a}}, 3^{\mathrm{a}}$ e $3^{\mathrm{a}}$; ROE: $4^{\mathrm{a}}$, $6^{\mathrm{a}}, 5^{\mathrm{a}}, 4^{\mathrm{a}}$ e $5^{\mathrm{a}}$; Capital de Giro: $5^{\mathrm{a}}, 4^{\mathrm{a}}, 4^{\mathrm{a}}, 5^{\mathrm{a}}$ e $6^{\mathrm{a}}$; Fluxo de Caixa: $6^{\mathrm{a}}, 5^{\mathrm{a}}, 6^{\mathrm{a}}, 6^{\mathrm{a}}$ e $8^{\mathrm{a}}$; Endividamento: $7^{\mathrm{a}}, 8^{\mathrm{a}}, 7^{\mathrm{a}}, 7^{\mathrm{a}}$ e $7^{\mathrm{a}}$; ROA: $8^{\mathrm{a}}, 7^{\mathrm{a}}, 8^{\mathrm{a}}, 8^{\mathrm{a}}$ e $1^{\mathrm{a}}$.

Este ranking demonstra que os indicadores liquidez geral, retorno do investimento e liquidez corrente são os que possuem maior informação econômica financeira sobre as empresas em análise. Estes indicadores são considerados também os principais oponentes no cenário da teoria dos jogos para as empresas, pois quando não apresentarem bons valores, irão comprometer a situação econômica financeira das empresas e sua representatividade no mercado.

Para analisar a alternância das posições dos indicadores econômicos financeiros no período analisado, a tabela 5 apresenta a correlação de Kendall.

Tabela 5 - Correlação do ranking dos indicadores

\begin{tabular}{c|l|c|c|c|c|c}
\hline \multicolumn{2}{|c|}{2} & $\mathbf{2 0 0 8}$ & $\mathbf{2 0 0 9}$ & $\mathbf{2 0 1 0}$ & $\mathbf{2 0 1 1}$ & $\mathbf{2 0 1 2}$ \\
\hline \multirow{2}{*}{2008} & Correlações de coeficiente & 1,000 & $0,786^{* *}$ & $0,857^{* *}$ & $0,929^{* *}$ & 0,286 \\
\cline { 2 - 7 } & Sig. (2 extremidades) & - & 0,006 & 0,003 & 0,001 & 0,322 \\
\hline \multirow{2}{*}{2009} & Correlações de coeficiente & $0,786^{* *}$ & 1,000 & $0,786^{* *}$ & $0,714^{* *}$ & 0,214 \\
\cline { 2 - 7 } & Sig. (2 extremidades) & 0,006 & - & 0,006 & 0,013 & 0,458 \\
\hline \multirow{2}{*}{2010} & Correlações de coeficiente & $0,857^{* *}$ & $0,786^{* *}$ & 1,000 & $0,929^{* *}$ & 0,286 \\
\cline { 2 - 7 } & Sig. (2 extremidades) & 0,003 & 0,006 & - & 0,001 & 0,322 \\
\hline \multirow{2}{*}{2011} & Correlações de coeficiente & $0,929^{* *}$ & $0,714^{* *}$ & $0,929^{* *}$ & 1,000 & 0,357 \\
\cline { 2 - 7 } & Sig. (2 extremidades) & 0,001 & 0,013 & 0,001 & - & 0,216 \\
\hline \multirow{2}{*}{2012} & Correlações de coeficiente & 0,286 & 0,214 & 0,286 & 0,357 & 1,000 \\
\cline { 2 - 7 } & Sig. (2 extremidades) & 0,322 & 0,458 & 0,322 & 0,216 & - \\
\hline
\end{tabular}

**. A correlação é significativa no nível 0,01 (2 extremidades).

*. A correlação é significativa no nível 0,05 (2 extremidades).

Fonte: Dados da pesquisa 
Observa-se na tabela 5, que houve correlação forte nos períodos de 2008/2009, 2009/2010, 2010/2011 com significância ao nível de 5\%. Esta correlação indica que a alternância dos indicadores econômicos financeiros nestes períodos foi baixa nos rankings, pois as posições dos indicadores permaneceram constantes. Como evidenciado na tabela 4, os resultados confirmam estatisticamente que são os indicadores liquidez geral, retorno do investimento e liquidez corrente os principais oponentes das empresas em análise, por possuírem as informações econômicas financeiras mais relevantes das empresas e por apresentarem-se constantes nos rankings.

\section{CONSIDERAÇÕES FINAIS}

O objetivo deste estudo de verificar o ranking das empresas de telecomunicações e de seus indicadores econômicos financeiros utilizando a estratégia mista da teoria dos jogos foi alcançado, visto que foi possível analisar as posições que as empresas e os indicadores ocuparam nos rankings no período de 2008 a 2012.

Por meio da correlação de Kendall verificou-se a alternância de posições nos rankings. Percebeu-se que a correlação do ranking das empresas foi baixa, demonstrando grande alternância das posições nos anos analisados. Já no que se refere aos indicadores, à correlação apresentou-se forte e significante, pois houve pouca alteração destes nas posições do ranking.

Os indicadores liquidez geral, retorno do investimento e liquidez corrente foram os que se mantiveram nas primeiras colocações do ranking no período analisado, representando os principais oponentes das empresas, uma vez que possuem importantes informações econômicas financeiras, que podem comprometer seu desempenho no mercado, ao não possuírem bons valores.

Sugere-se para pesquisas futuras, analisar situações empresariais utilizando a teoria dos jogos com indicadores sociais, ambientais e de mercado, a fim de verificar os principais indicadores considerados oponentes das empresas na visão desta teoria.

\section{REFERÊNCIAS}

ABBADE, E. B. Análise de Alianças Estratégicas so

b a ótica da Teoria dos Jogos. Revista de Administração da Unimep, 7(3), 24-45, 2009.

ALENCAR, A. G.; OMAKI, E. T.; DRUMOND JUNIOR, M. A.; PERES, I. M. Um Olhar da Teoria dos Jogos Sobre a Fusão da Sadia com a Perdigão. Anais do Encontro da Associação Nacional de Pós-graduação e Pesquisa em Administração, Rio de Janeiro, RJ, Brasil, 2010.

BASTOS, D. D.; NAKAMURA, W. T.; DAVID, M.; ROTTA, U. A. S. A relação entre o retorno das ações e as métricas de desempenho: evidências empíricas para as companhias abertas no Brasil. REGE Revista de Gestão, 16(3), 65-79, 2009.

BEZERRA, F. A.; CORRAR, L. J. Utilização da análise fatorial na identificação dos principais indicadores para avaliação do desempenho financeiro: uma aplicação nas empresas de seguros. Revista de Contabilidade e Finanças-USP, São Paulo, (42), 50-62, 2006.

BLACKWELL, D.; GIRSHICK, M. Theory of games and statistical decisions. John Wiley \& Sons, 1954.

BORTOLUZZI, S. C.; ENSSLIN, S. R.; LYRIO, L.; VASCONCELLOS, M.; ENSSLIN, L. Avaliação de desempenho econômico-financeiro: uma proposta de integração de indicadores contábeis tradicionais por meio da metodologia multicritério de apoio à decisão construtivista (MCDA-C). Revista Alcance, 18(2), 2011.

CHARNES, A. Constrained games and linear programming. Proceeding of the National Academy of Science, 39, 639-641, 1963. 
DAVIS, M. Teoria dos jogos: uma introdução não-técnica. Prefácio de Oskar Morgenstern, tradução de Leonidas Hegenberg e Otanny Silveira da Mota. São Paulo: Cultrix, 1973.

DRESHER, M.; SHAPLEY, L.; TUCKER, A. W. Advances in game theory. Annals of Mathematical Studies, 39.Princeton University Press, 1957.

DRESHER, M.; TUCKER, A. W.; WOLFE, P. Contributions to the theory of game. Annals of Mathematical Studies, 52. Princeton University Press, 1964.

FERNÁNDEZ, F. R; MONROY, L.; PUERTO, J. Multicriteria goal games. Journal of optimization theory and applications, 99 (2), 403-421, 1998.

FIANI, R. Teoria dos jogos. 2 ed. Rio de Janeiro: Elsevier Brasil, 2006.

GALE, D. The theory of linear economic models. New York: McGraw-Hill, 1960.

GROSSMAN, S. I. Aplicaciones de algebra lineal. Mexico:McGraw-Hill, 1992.

HARSANY, J. C. Rational behavior and bargaining equilibrium in games and social situations. New York: Cambridge University Press, 1977.

HEIN, N.; KROENKE, A.; FARIA, T. M. B. Teoria dos Jogos em Sistemas de Informações Interdependentes. In: XX EPIO. Escuela de Perfeccionamento em Investigación Operativa, Buenos Aires, Argentina, 2009.

KAKUTANI, S. Concrete representation of abstract (M)-spaces (A characterization of the space of continuous functions). Annals of Mathematics, 994-1024, 1941.

KARLIN, S. Mathematical methods and theory in games. Programming and Economics, v. 2, New York: Addison-Wesley, 1959.

KREUZBERG, F. Indicadores econômicos versus indicadores sociais: uma análise de empresas listadas na BM\&F Bovespa por meio da Teoria dos Jogos. Dissertação do Programa de Pós-Graduação em Ciências Contábeis do Centro de Ciências Sociais Aplicadas da Universidade Regional de Blumenau, Santa Catarina, Brasil, 2013.

KROENKE, A.; HEIN, N.; RODRIGUES JUNIOR, M.M; HINSCHING, L. C.; WILHELM, V. E. Teoria dos Jogos abordados em artigos e eventos científicos na área de administração e contabilidade. Anais do XIX Erematsul, Santa Maria, RS, Brasil, 2013.

KUHN, H. W.; TUCKER, A. W. Contributions to the theory of games. Annals of Mathematical Studies, n. 24 and 28. Princeton University Press, 1950.

KUHN, H. W.; TUCKER, A. W. Contributions to the theory of games. Annals of Mathematical Studies, v 24 and 28. Princeton University Press, 1953.

LINS, M. P. E.; CALÔBA, G. M. Programação Linear: com aplicações em teoria dos jogos e avaliação de desempenho. Rio de Janeiro: Editora Interciência, 2006.

LOZANO, R. Addressing stakeholders and better contributing to sustainability through game theory. Journal of Corporate Citizenship, 43, 45-62, 2011.

LUCE, D.; RAIFFA, H. Games and decisions. New York: John Wiley ans Sons, 1957.

MC KINSEY, J. C. C. Introduction to the theory of games. New York: McGraw-Hill, 1952.

MCCAIN, R. Cooperative games and cooperative organizations. The Journal of SocioEconomics, 37, 2155-2167, 2008.

MYERSON, R. B. Game theory. Harvard university press, 2013.

OWEN, G. Game theory. San Diego:W.B. Sauders, 1968. 
PARTHASARATHY, T.; RAGHAVAN, T. E. S. Some topics in two-person games. American Elsevier, 1971.

RAPOPORT, A. Two-person game theory: the essential ideas. Michigan: University of Michigan Press, 1966.

RAPOPORT, A. Person game theory: concepts and aplications. Michigan: University of Michigan Press, 1970.

SANVICENTE, A. Z.; MINARDI, A. M. A. F. Identificação de indicadores contábeis significativos para a previsão de concordata de empresas. Instituto Brasileiro de Mercado de Capitais, Working Paper, 1998.

SHUBIK, M. Strategy and Market structure: competition, oligopoly and the theory of games. John Wiley \& Sons, 1959.

SHUBIK, M. Market structure and behavior. Massachusetts: Harward University Press, 1980.

SILVA, V.A.; CORDEIRO FILHO, J. B. Análise Crítica da produção científica em contabilidade, utilizando a Teoria dos Jogos, no suporte à tomada de decisões, 2010. Disponível

http://www.contabeis.ufba.br/Site/arquivos/Editor/file/Mestrado/Artigos/2010/AN\%C3\%81LI SE\%20CRITICA\%20DA\%20PRODU\%C3\%87\%C3\%830\%20CIENT\%C3\%8DFICA\%20E M\% 20\% 20\%20CONTABILIDADE, \%20UTILIZANDO\%20A\%20TEORIA\%20DOS\%20JO GOS $\% 20 \% 20, \% 20$ NO $\% 20$ SUPORTE $\% 20 \% \mathrm{C} 3 \% 80 \% 20 \mathrm{TOMADA} \% 20 \mathrm{DE} \% 20 \mathrm{DECIS} \% \mathrm{C} 3$ \%95ES.pdf. Acesso em: 20 fevereiro 2014.

SINGLETON, R. R.; TYNDALL, W. F. Games and programs : mathematics for modeling. San Francisco, CA: Freeman, 327, 1974.

SOUZA, Á. A. A teoria dos jogos e as ciências sociais. 176 f. Dissertação (Mestrado em Ciencias Sociais) Faculdade de Filosofia e Ciências da Universidade Estadual Paulista Júlio de Mesquita Filho. Marília: UNESP, 2003.

TUCKER, A. W.; LUCE, D. Contributions to the theory of games. Annals of Mathematical Studies, 4 (40). Princeton University Press, 1959.

VON NEUMANN, J. Zur theorie der gesellshaftsspiele. 296-320, 1928.

VON NEUMANN, J.; MORGENSTERN, O. Theory of games and economic behavior. Princeton: Princeton University Press, 1944.

VON NEUMANN, J.; MORGENSTERN, O. Theory of games and economic behavior. Princeton: Princeton University Press, 1953.

ZELENY, M. Multiple criteria decision making. New Yor: McGraw-Hill, 1982. 